\title{
Comparisons of SDR performance of gibbons and three species of New World monkeys on a spatial task 1
}

ROBERT L. GOSSETTE, Comparative Animal Behavioral Laboratory, Hofstra University, Hempstead, Long Island, N.Y. 11550

The successive discrimination reversal (SDR) performances of four gibbons were obtained on a spatial task and compared with performances of squirrel, owl, and capuchin monkeys previously obtained on the same task. The gibbon's performance was notably inferior to that of the capuchin and rather more closely resembled that of the owls and squirrels. The value of interspecies SDR performance comparisons in establishing a behavior taxonomy is discussed.

In the comparative analysis of primate learning, the most popular and extensively employed methodology has been that of learning set (LS). Comparisons of LS performances of a number of species of New World and Old World monkeys, as well as the great apes, have given rise to the general conclusion that relative level of success, or efficiency, of problem solving is highly correlated with taxonomic and/or phyletic separation (Rumbaugh, 1968; Warren, 1965). However, due to the intrinsic difficulty of typical LS problem sets, and in particular the distinctive procedure that defines LS methodology, the sensitivity of LS to interspecies differences would appear to be sharply reduced among the inframammalian species and even among many mammalian species. Thus, to provide a more comprehensive analysis of vertebrate learning, across broader reaches of phyletic or taxonomic separation, an alternative strategy would appear desirable.

One important alternative, sometimes viewed as a special instance of $L S$, is successive discrimination reversal (SDR) methodology. In this methodology, the stimulus sets constituting the successive problems remain constant, as contrasted with LS stimulus sets, with only a periodic change in the reinforcement contingencies. In a series of preliminary investigations in this laboratory with a number of different avian and mammalian species, interspecies SDR performance differences have been isolated from intraclass comparisons that are consistent with interspecies "taxonomic distance" (Gossette, Gossette, \& Riddell, 1966; Gossette, 1966; Gossette \& Slonim, 1968). The author has suggested elsewhere (Gossette, 1969a, b) that the major sources of the sensitivity of SDR to interspecies differences arise from (1) a maximal opportunity for the occurrence of negative transfer under conditions of (2) inconstancy of reinforcement. These two factors are typically not controlled experimentally in LS methodology; nor are their effects isolated behaviorally in the usual LS analysis of interspecies performance differences. LS procedures, containing a randomized sequence of both complete and partial reversal problems, as well as nonreversal problems, appear to be less efficient for the screening of interspecies performance differences than SDR procedures. It would be of considerable interest to learn whether or not the previously reported interspecies differences in LS error scores were largely contributed by those problems that constitute a reversal, either partial or complete, of a preceding problem's reinforcement contingencies. However, one problem presented by SDR methodology as typically employed is that its sensitivity to interspecies differences may be limited, may reach a ceiling, among "higher" primates by virtue of the simplicity of the task. SDR performance of capuchin monkeys, for example, quickly reaches a one-error solution on a spatial task (Gossette \& Inman, 1966).

As a further step in the exploration of the usefulness of SDR in the calibration of interspecies performance differences among primates, it would be of some interest to compare performances of one of the great apes with those of the phyletically inferior New World monkeys, for whom data alreadv exists.

\section{SUBJECTS}

Three female and one male adolescent white-handed gibbons (Hylobates lars) were employed in the present experiment. One male and one female were acquired at approximately 8 to 10 months of age and have been maintained in the laboratory for about 4 years. The other two females were acquired approximately 1 to $1 \frac{1 / 2}{2}$ years ago and appear to be approximately at the same developmental level as the first two Ss. All Ss have been maintained in individual caging, with a diet of Purina Monkey Chow, abundantly supplemented with bananas, fresh fruits and vegetables, and vitamins. The original male and female in the colony have received extensive habituation to human handling over the course of 4 years. The two recently obtained females have received extensive handling in this laboratory for the past year. Their prior history is generally unknown. However, all four Ss were naive with respect to the kinds of apparatus employed in learning experiments. APPARATUS

The apparatus employed in SDR training was a formboard box previously used in the study of SDR performances of squirrel, owl, and capuchin monkeys (Gossette \& Slonim, 1969).

\section{PROCEDURE}

Each $S$ was habituated to confinement in the apparatus for about 3 weeks. During this period, each $S$ was trained by successive approximation to dislodge stimulus blocks to obtain approximately one-quarter of a large Concord grape from the underlying foodwells. When response to the two white round stimulus blocks was well established, with equal training given to both block positions, a position habit was established, with reinforcement provided for responding to the nonpreferred position. A criterion procedure was employed, with daily sessions of 20 noncorrection trials, until two or fewer errors were recorded on a given day's session. At that point, the reinforcement contingencies were reversed and training proceeded as before. In this fashion, a total of 19 reversals was obtained.

Previous work (Gossette \& Feldman, 1968; Gossette, 1969a, b) has established that motivational and incentive levels are important determinants of SDR performance among both birds and mammals. In these investigations, low drive levels, e.g., $5 \%$ to $10 \%$ loss of ad lib body weights, tended to facilitate error reduction as contrasted with $S$ s trained at high drive levels, e.g., $25 \%$ to $30 \%$ loss. To facilitate comparison of gibbon performance with that of owl, squirrel, and capuchin monkeys, previously reported, a low-drive condition was assigned to the gibbons. However, due to aversiveness of weighting procedures to all four gibbons, and the absence of alternative procedures, it was decided to eliminate weight loss as a control for drive level. Instead, each $S$ was tested each aftemoon immediately prior to its usual feeding period. The food given each day was the same in amount and variety that had been customary prior to experimentation. No restriction was placed upon the time permitted to eat the daily ration. Typically, all food had been consumed by the next morning. Thus it seems reasonable to believe that drive level for all Ss was minimal. The choice of incentive level was dictated largely by intuitive grounds. Since one-eighth of a grape had been emploved with the much 


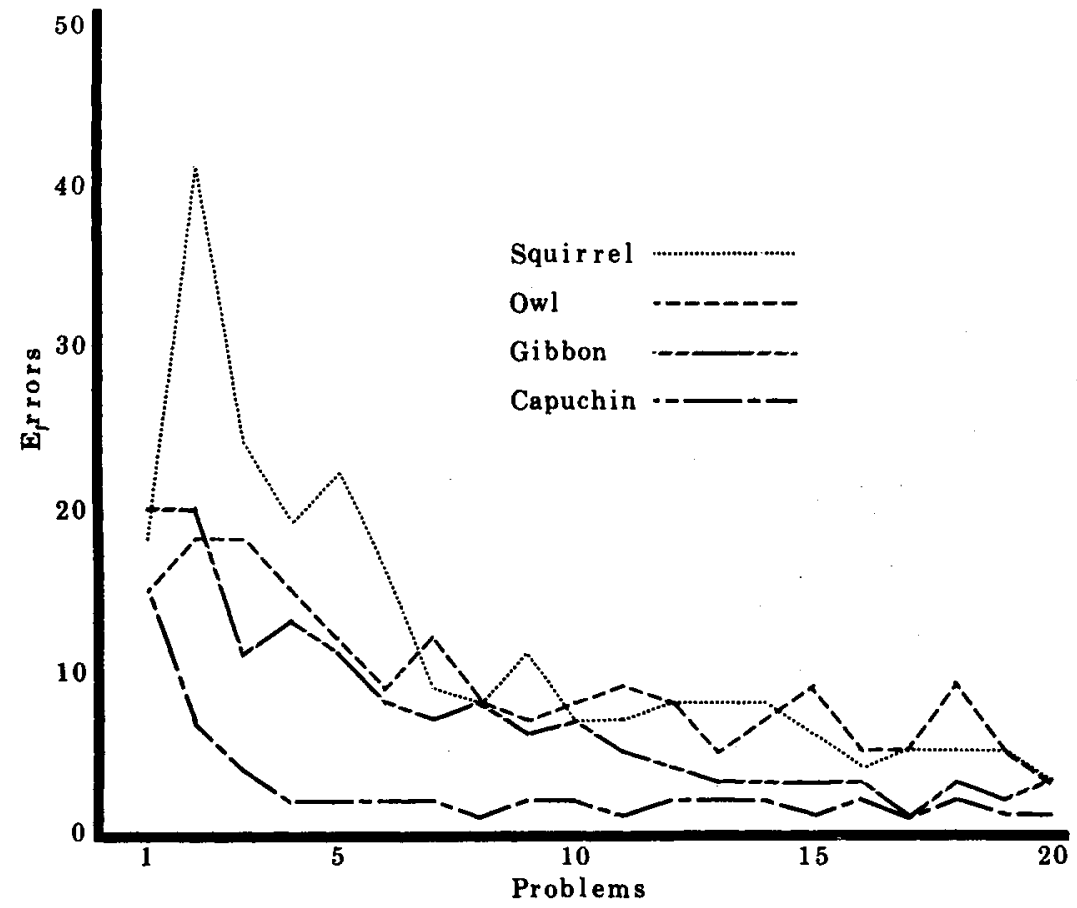

smaller New World monkeys, it was decided to double the size of the grape reward given to the gibbons. A larger reward was impossible due to the size of the foodwells.

RESULTS AND DISCUSSION

The principle SDR response selected for analysis was the mean total errors across all 20 problems. Table 1 presents the range and mean of total errors for all four groups. Analysis of these means by Kiuskal-Wallis test revealed that interspecies performance differences were significant $(p<.05)$. Examination of individual scores indicates an appreciable overlap of performance of the owls and squirrel monkeys as previously reported. These two groups also clearly produced the largest error scores. The capuchins produced the lowest errors, with no overlap with any of the other groups. While the gibbon mean error score was the second lowest, there was a slight overlap with owl-squirrel groups, with one gibbon producing an error score greater than the lowest scorer among the owl-squirrel Ss. Figure 1 presents mean errors across problems for all groups.

The most striking feature of the present comparisons is the relative inferiority of the gibbons. Interestingly, a similar finding has been reported by Rumbaugh (1968) employing LS methodology. He found that gibbon performance on LS, as measured by

percentage of Trial 2 correct responses, was clearly inferior, as might be expected from phyletic considerations, as well as comparison of brain indices, to that of gorillas, chimpanzees, and orangutans over a wide range of age levels. However, gibbon performance was also uniformly inferior to that of macaques and even that of squirrel monkeys. While the age range of the macaques tested was appreciable, 2 to 9 years, the squirrel monkeys were at least 9 years or older, considerably older than most squirrel monkeys employed in such research.

While neither the variety nor the range of taxonomic separation of primate species that have been sampled by LS methodology have been equaled as yet with SDR procedures, it would appear that the latter is as promising as LS in generating interspecies performance differences that are taxonomically meaningful. While both methodologies yield the phenomenon commonly called "learning to learn," or a progressive reduction in errors across problems, SDR typically reveals an initial negative transfer, absent most notably in the capuchin data described above. Analysis of SDR performances among birds has shown that it is variation in magnitude of this negative transfer that is the principle source of performance differences among species differing in degree of taxonomic and/or Table 1

Range and Mean of Total Errors Across 20 Spatial Reversal Problems

\begin{tabular}{lcccc}
\hline & Owl & Squirrel & Capuchin & Gibbon \\
\hline Mean & 187.0 & 221.1 & 54.2 & 136.3 \\
Range & $120-234$ & $150-330$ & $30-75$ & $100-203$ \\
\hline
\end{tabular}

Fig. 1. Mean error for gibbon and three species of New World monkeys across 20 spatial SDR problems.

phyletic separation (Gossette, in press). To some extent this is also true of the primate comparisons described above; both owl and squirrel monkeys displayed the greatest negative transfer. If further sampling of primate species with SDR procedures, paralleling that already reported with LS, demonstrates that SDR generates interspecies performance differences as distinctive as those arising from LS analysis, SDR analysis would appear to possess at least two advantages in the analysis of vertebrate learning. For one, SDR analysis can be extended over a far greater range of taxonomic separation, from mammals to fish, possibly extending to certain groups of invertebrates. Thus more general, comprehensive formulations of those dimensions that underlie interspecies variation in learning may be feasible. Secondly, the relative simplicity of the stimulus controls provided by SDR procedures, as contrasted with the highly heterogeneous and variable controls typical of LS methodology, would appear to greatly simplify the task of experimentally isolating these dimensions. At present three different attempts to identify these dimensions have been proposed: the retention decrement (Gonzalez et al, 1967), the differential extinction (Gossette, 1968), and the attention (Machintosh, 1969) hypotheses.

The similarity of the owl and squirrel monkey data suggests a provocative question. The taxonomic distance hypothesis (Gossette, in press) that has emerged from a series of SDR analyses conducted by the author with a variety of different mammalian and avian species states that similarity of SDR performance across species is a function of interspecies taxonomic distance. That is, closely related species will display more similar error functions than will more distantly related species. Traditional taxonomical practice has been to group the squirrel monkeys with the capuchins, in the subfamily Cebinae (Simpson, 1945). Yet other writers (Sanderson, 1957) have argued that many points of similarity between the owl and squirrel monkeys require the reassignment of the squirrel to reflect the similarity. To the extent that similarity of patterns of learned behavior may be useful diagnostic clues in taxonomy, the similarity of SDR performance lends support to such realignment.

\section{REFERENCES}

GONZALEZ, R. C., BEHREND, E., \& BITTERMAN, M. E. Reversal learning and 
forgetting in bird and fish. Science, 1967, 158, 519-521.

GOSSETTE, R. Comparisons of successive discrimination reversal (SDR) performances across fourteen different avian and mammalian species. American Zoologist, 6, 157, No. 4, November 1966 (Abstract).

GOSSETTE, R. An examination of the retention decrement explanation of comparative successive discrimination reversal learning by birds and mammals. Perceptual \& Motor Skills, $1968,27,1147-1152$.

GOSSETTE, R. The role of motivational and incentive levels in the control of successive discrimination reversal (SDR) performance in comparative analysis. Perceptual \& Motor Skills, 1969a, 28, 63-68.

GOSSETTE, $R$. Variation in magnitude of negative transfer on successive discrimination reversal (SDR) tasks across species. Perceptual \& Motor Skills, 1969b, 29, 803-811.

GOSSETTE, R. A note on the calibration of inter-species successive discrimination reversal (SDR) performance differences: Qualitative vs quantitative scaling. Perceptual \& Motor Skills, in press.

GOSSETTE, R., \& FELDMAN, J. An examination of the sensitivity of successive discrimination reversal (SDR) measures to differences in motivational level with squirrel monkeys. Psychonomic Science, 1968, 11, 157-158.

GOSSETTE, R., GOSSETTE, M., \& RIDDELL, W. Comparisons of successive discrimination reversal performances among closely and remotely related species. Animal Behaviour, $1966,14,560-564$.
GOSSETTE, R., \& INMAN, N. Comparisons of spatial successive discrimination reversal performances of two groups of New World monkeys. Perceptual \& Motor Skills, 1966, 23 169-170.

GOSSETTE, R., \& SLONIM, P. Spatial SDR performances across three species of New World monkeys. Psychonomic Science, 1969 $14,222-223$.

MACKINTOSH, N. J. Comparative studies of reversal and probability learning: Rats, birds, and fish. In N. S. Sutherland and R. Gilbert (Eds.), Animal discrimination learning. London: Academic Press, 1969. Pp. 137-162.

RUMBAUGH, D. The learning and sensory capacities of the squirrel monkey in phylogenetic perspective. In L. Rosenblum and R. Cooper (Eds.), The squirrel monkey. New York: Academic Press, 1968. Pp. 256-317.

SANDERSON, I. The monkey kingdom. Garden City, N.Y: Hanover House, 1957.

SIMPSON, G. G. The principles of classification and a classification of mammals. Bulletin of the American Museum of Natural History, 1945,85

WARREN, J. M. Comparative psychology of learning. In Annual review of psychology. Vol. 16. Palo Alto, Calif: Annual Reviews, Inc., 1965. Pp. 95-118.

\section{NOTE}

1. Supported in part by Grant No. MH 16293-01 awarded to the author by NIMH.

1961). Thirsts induced by hypertonic and hyperoncotic treatments or hemorrhage are additive to potentiate drinking (Corbit, 1968; Fitzsimons \& Oatley, 1968).

Since (1) feeding and drinking are reciprocally related, and (2) hypertonic and hyperoncotic treatments add to potentiate drinking, it is expected that these treatments also add to inhibit feeding. This study investigated hypertonic saline and hyperoncotic colloid treatments on feeding and drinking.

SUBJECTS AND APPARATUS

The Ss were 24 female Wistar rats, about 180 days old, weighing from 210 to $291 \mathrm{~g}$ They were housed individually in a constantly illuminated laboratory at a temperature of $22^{\circ}-23^{\circ} \mathrm{C}$; humidity varied from $38 \%$ to $42 \%$. A $100-\mathrm{ml}$ graduated tube with metal tip was mounted on each home cage and used to measure water intake. Food intake was measured by use of a $250 \mathrm{ml}$ glass beaker fastened at a corner of each cage. Powdered Purina Lab Chow was introduced into the beaker for feeding. Food spillage was minimal because rats ate with their heads inside the beakers. Food intake was read to the nearest $0.1 \mathrm{~g}$ and water intake to the nearest $1 \mathrm{ml}$.

\section{PROCEDURE}

The Ss were adapted to a schedule of 1-day total deprivation and 1-day feeding-drinking for 8 days. On feeding-drinking days, food alone was presented for $1 \mathrm{~h}$ followed by water only for $1 \mathrm{~h}$ before both food and water were given for $22 \mathrm{~h}$. By the eighth day of the adaptation period, body weight, food intake, and water intake had become stable and the experiment was begun. Ss were divided into four groups of six Ss each, equated according to their $1-h$ food intake measures.

\section{Experiment 1}

All Ss were deprived of food and water for $24 \mathrm{~h}$ and were given food only for $1 \mathrm{~h}$ to measure intake. Six hours before the

\section{SIGMUND HSIAO, University of Arizona Tucson, Ariz. 85721}

Rats were given injections of a hyperoncotic colloid and/or a hypertonic saline solution. Six hours after the oncotic load and $30 \mathrm{~min}$ after the osmotic load, food alone was presented for $1 \mathrm{~h}$. Rats had been deprived of both food and water for $24 \mathrm{~h}$ when food was presented. Six days later, the same Ss were given the same treatments and given water only for $1 \mathrm{~h}$. Results showed that both treatments additively inhibited feeding and potentiated drinking. Feeding and drinking as influenced by the colloid and the saline treatments are reciprocally related.

Thirst and hunger are closely related. In ad lib feeding conditions there is a precise temporal relation between feeding and drinking (Kissileff, 1969) and a positive correlation between the size of food and of water intake related to a meal (Fitzsimons \& Le Magnen, 1969). Limiting food intake reduces drinking proportionally to the amount eaten (Hsiao \& Pertsulakes ${ }^{2}$ ), and rationing water or deterring water intake by quinine reduces food intake proportionally to the amount drunk (Collier \& Levitsky, 1967; Hsiao \& Lloyd, 1969). Water injection in thirsty rats reduces drinking but increases feeding proportionally to the amount injected (Hsiao \& Trankina, 1969). Thus, thirst inhibits feeding, feeding induces drinking, and, conversely, reduction in thirst potentiates feeding in rats.

Increase in drinking can be induced by injections of hypertonic saline (e.g., Corbit 1969), hyperoncotic colloid (Stricker, 1966), or by hemorrhage (Fitzsimons, food presentation, each $S$ in Groups 1 and 3 was given a subcutaneous (backskin behind the neck) injection of a hyperoncotic colloid which was $5 \mathrm{ml}$ of $10 \%(\mathrm{w} / \mathrm{v})$ polyethylene glycol (PG) (molecular weight $=20,000$ ) dissolved in isotonic saline. Groups 2 and 4 were similarly injected with $5 \mathrm{ml}$ of isotonic saline vehicle. Ss were lightly etherized before these injections. Thirty minutes before the food presentation, each $S$ in Groups 1 and 2 was given an intraperitoneal injection of $3 \mathrm{ml}$ of $1 \mathrm{M}$ $\mathrm{NaCl}$ solution. Groups 3 and 4 were given sham injection with needle puncture only. This was a 2 by 2 factorial design in which Group 1 received both hyperoncotic and hyperosmotic loads, Group 2 received hyperosmotic load only, Group 3 received hyperoncotic load only, and Group 4 